\title{
Providencia stuartii
}

National Cancer Institute

\section{Source}

National Cancer Institute. Providencia stuartii. NCI Thesaurus. Code C86695.

A species of aerobic, Gram negative, rod shaped bacteria in the phylum Proteobacteria.

This species is motile, produces acid from galactose, adonitol, inositol, and trehalose. P. stuartii is an opportunistic pathogen found in multiple animal reservoirs, soil, water, and sewage that can cause catheterization related urinary tract infections or diarrhea. 